\title{
Hair Follicle Nevus Located on the Neck: Comparison with Accessory Tragus, Cervical Chondrocutaneous Branchial Remnants and Trichofolliculoma
}

\author{
Ji Su Lee, Ji Hoon Yang, Hyunsun Park, Hyun-Sun Yoon, Soyun Cho \\ Department of Dermatology, SMG-SNU Boramae Medical Center, Seoul, Korea
}

\begin{abstract}
Hair follicle nevus (HFN) is a rare, benign, follicular hamartoma that most frequently presents as a congenital nodule on the face. We experienced a rare case of HFN on the neck of a 14-year-old boy and performed a pilot immunohistochemical study with cytokeratin 19 (CK19) to compare the staining pattern of hair follicles in HFN and its differential diagnoses, accessory tragus, cervical chondrocutaneous branchial remnants (CCBR) and trichofolliculoma. With hematoxylin and eosin stain, HFN showed numerous tiny hair follicles in the dermis with several sebaceous and eccrine glands, and perifollicular fibrous thickening. With CK19 stain, some hair follicles in HFN and CCBR showed positive expression, a few hair follicles in accessory tragus showed weak expression, and no hair follicles in trichofolliculoma showed expression. The present report supports the view that HFN, accessory tragus and CCBR are within the same spectrum of hamartomas. (Ann Dermatol 31(6) 662 665, 2019)
\end{abstract}

\section{-Keywords-}

Accessory tragus, Cervical chondrocutaneous branchial remnants, Cytokeratin 19, Hair follicle nevus, Trichofolliculoma

Received May 16, 2018, Revised September 3, 2018, Accepted for publication September 19, 2018

Corresponding author: Soyun Cho, Department of Dermatology, SMG-SNU Boramae Medical Center, 20 Boramae-ro 5-gil, Dongjak-gu, Seoul 07061, Korea. Tel: 82-2-870-2381, Fax: 82-2-870-3866, E-mail: sycho@snu.ac.kr ORCID: https://orcid.org/0000-0003-2468-485X

This is an Open Access article distributed under the terms of the Creative Commons Attribution Non-Commercial License (http://creativecommons. org/licenses/by-nc/4.0) which permits unrestricted non-commercial use, distribution, and reproduction in any medium, provided the original work is properly cited.

Copyright (c) The Korean Dermatological Association and The Korean Society for Investigative Dermatology

\section{INTRODUCTION}

Hair follicle nevus (HFN) is a rare, benign, follicular hamartoma that usually presents as a congenital, asymptomatic nodule on the face. Differential diagnoses of HFN include accessory tragus, cervical chondrocutaneous branchial remnants (CCBR) and trichofolliculoma, but recently there has been a debate regarding whether they are on a spectrum of the same entity ${ }^{1-4}$. Here we report the case of a HFN which presented as a solitary papule on the neck. In order to investigate and compare the characteristics of hair follicles in HFN and its differential diagnoses, a pilot immunohistochemical study with cytokeratin 19 (CK19, a marker for hair follicle stem cell) was performed in the presented case and each case of accessory tragus, CCBR, and trichofolliculoma. We received consent from the patient's parents about publishing all photographic materials.

\section{CASE REPORT}

A 14-year-old boy presented with a skin tag-like lesion on his right lower neck, and a red-colored papule on his right upper neck (Fig. 1A). The lower lesion was asymptomatic and had been present since birth. The upper lesion developed 1 month ago and bled frequently. The patient had been otherwise healthy with no known prenatal or developmental abnormalities or significant family history. Physical examination revealed a soft, pedunculated, skin-colored papule, $3 \mathrm{~mm}$ in diameter, on the slightly elevated surface on the right lower neck (Fig. 1B), and a soft, domeshaped, red-colored papule, $2 \mathrm{~mm}$ in diameter, on the right upper neck (Fig. 1A). Under the clinical impressions of skin tag and pyogenic granuloma, respectively, punch excision was performed for each lesion. Histopathologically, the lower skin-colored papule demonstrated a polypoid 

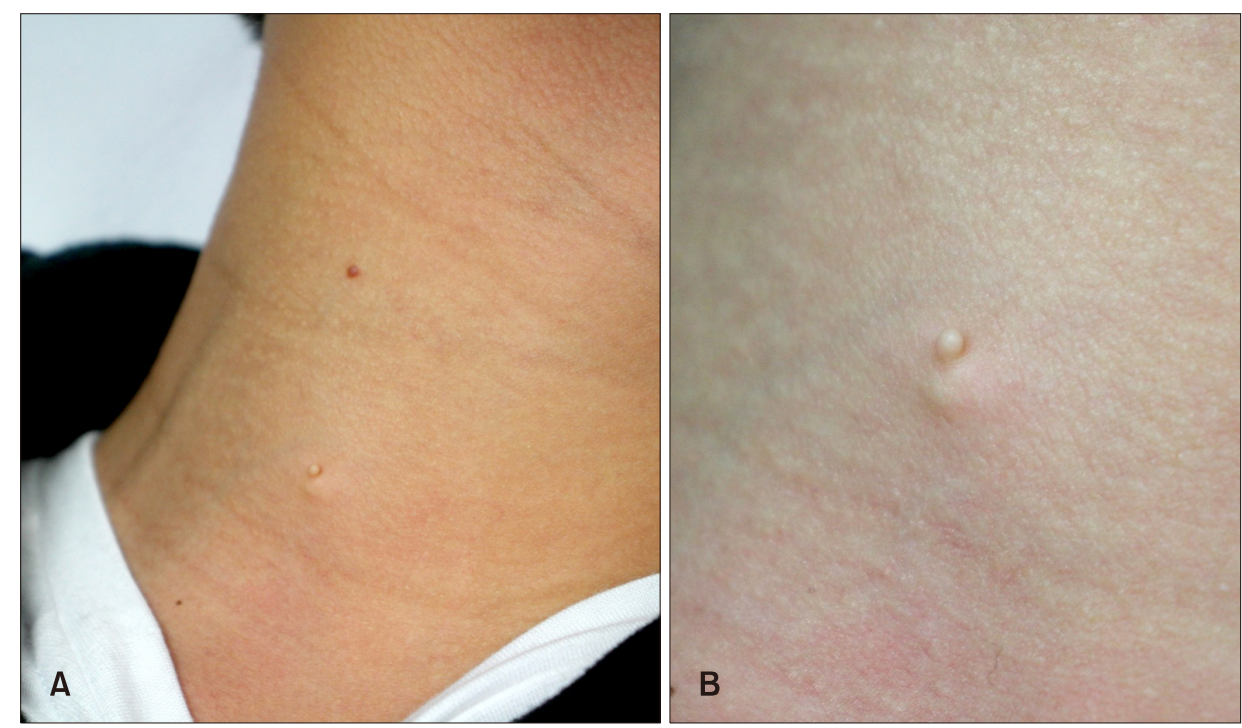

Fig. 1. Two separate papules on the right neck. (A) Asymptomatic and soft pedunculated skin-colored papule on the right lower neck and red-colored papule with bleeding tendency on the right upper neck. (B) Closer view of the lower fleshcolored lesion.
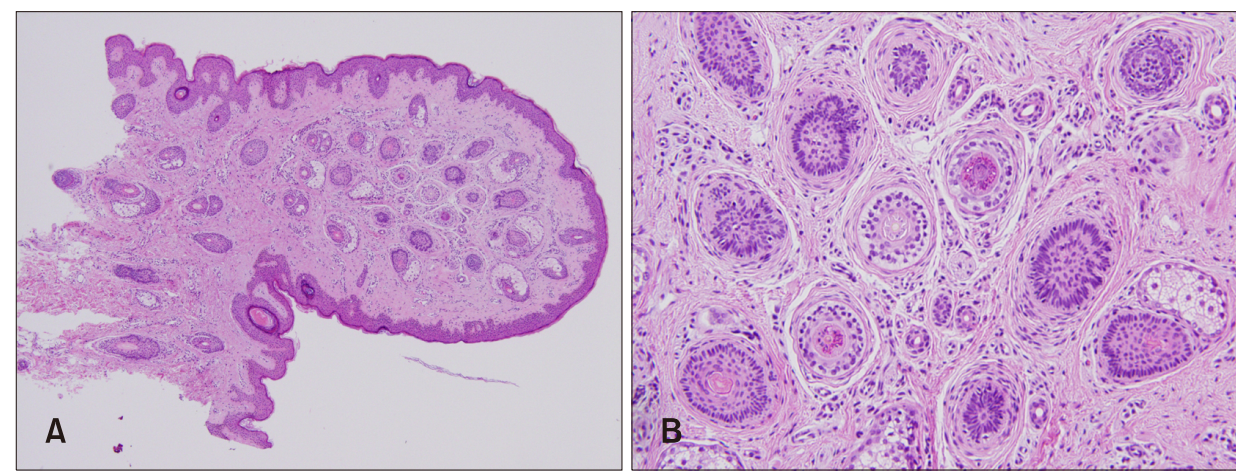

Fig. 2. Histopathology of the lower skin-colored papule. (A) Numerous vellus hair follicles are located in the dermis, and several sebaceous and eccrine glands are connected to the hair follicles $(\mathrm{H} \& \mathrm{E}, \times 40)$. (B) Perifollicular stroma shows fibrous thickening $(H \& E, \times 200)$. skin segment with mild epidermal hyperplasia and many vellus hair follicles in the dermis (Fig. 2A). Several sebaceous and eccrine glands connected with the hair follicle were also seen (Fig. 2A), and perifollicular stroma showed fibrous thickening (Fig. 2B). Serial sections did not reveal any cartilage, cystic follicular structures in the stroma, or prominent connective tissue framework in the subcutaneous fat. The upper red papule showed an exophytic lesion with lobular proliferation of capillaries, consistent with pyogenic granuloma. Based on the clinical and pathologic findings, diagnoses of HFN and pyogenic granuloma were made, respectively.

With CK19 (1:200, M0888; DAKO, Glostrup, Denmark) immunohistochemical stainining, follicular epithelium of some of the follicles in HFN and CCBR demonstrated positive expression (Fig. 3A, B). In accessory tragus, virtually all hair follicles showed negative expression except for a few follicles which showed weak expression of CK19 focally (Fig. 3C). In contrast, trichofolliculoma demonstrated no expression of CK19 in the hair follicles at all (Fig. 3D).

\section{DISCUSSION}

HFN is a rare, benign, follicular hamartoma typically presenting as a solitary, asymptomatic, skin-colored papule or nodule on the face at birth ${ }^{1,5}$. Histologically, HFN is characterized by proliferation of vellus hair follicles with perifollicular fibrous thickening occasionally surrounded by a cellular stroma. Sebaceous and eccrine glands and smooth muscle fibers are occasionally seen ${ }^{1,6,7}$.

The most difficult differential diagnoses of HFN include accessory tragus, CCBR, and trichofolliculoma'. They show similar clinical and histological features to HFN (Table 1) ${ }^{1,3-9}$. Some authors have suggested histologic differential points between HFN and accessory tragus; cartilaginous components in the stroma, prominent connective tissue framework in the subcutaneous fat, and abundant subcutaneous fat favors accessory tragus over HFN (Fig. 3B, inset) ${ }^{1}$. However, other authors have stated that HFN and accessory tragus are on the same spectrum ${ }^{1}$. Accessory tragus is considered to be of branchial arch origin. Typical preauricular lesion originates from the first branchial arch, and cervical 

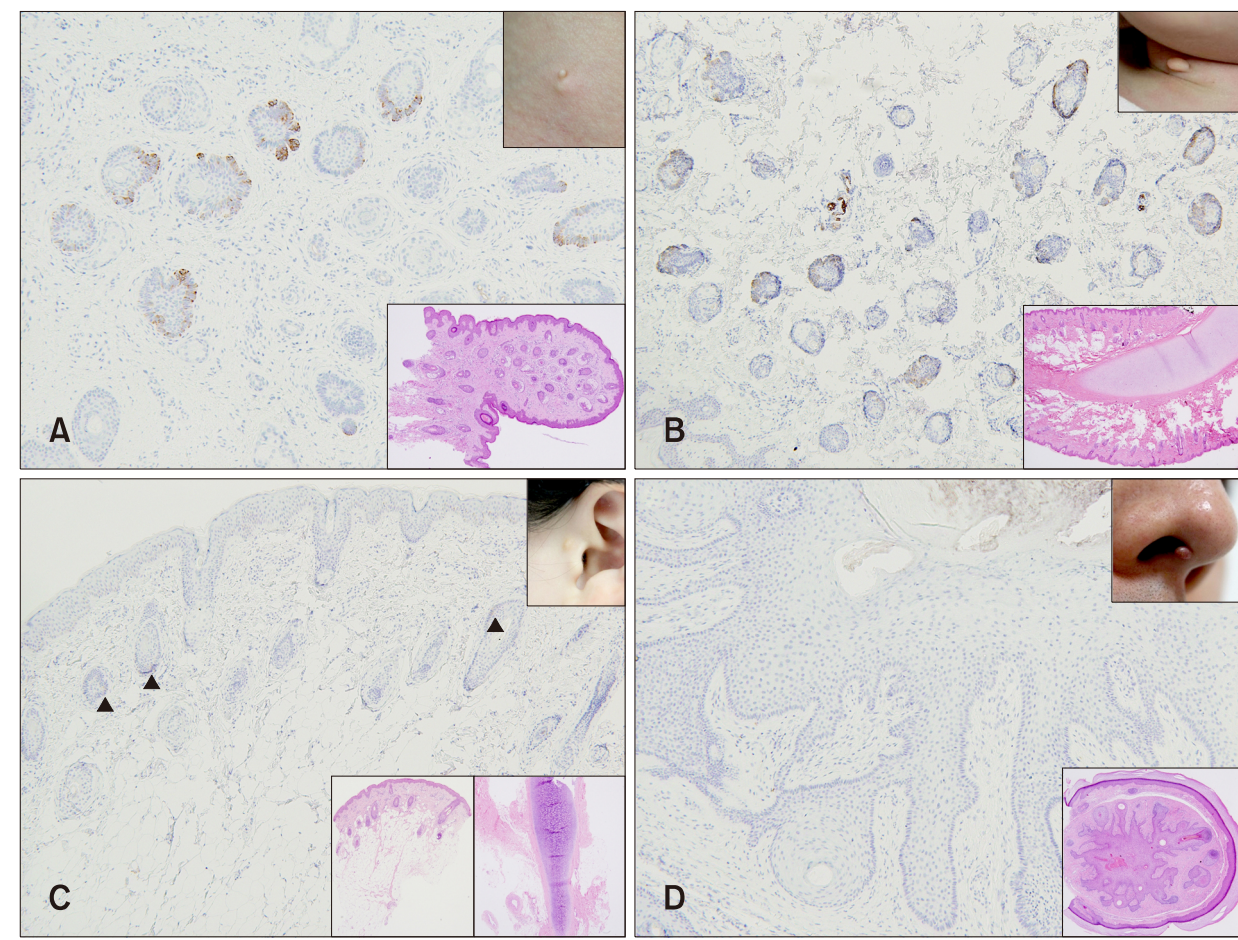

Fig. 3. Cytokeratin 19 (CK19) expression in the hair follicles in hair follicle nevus (HFN) (14-year-old), accessory tragus (8-year-old), cervical chondrocutaneous branchial remnants (CCBR) (24-month-old), and trichofolliculoma (37-year-old) (upper right insets, clinical pictures; lower right insets, hematoxylin and eosin-stained pictures in 40 fold magnification). (A, B) Some hair follicles in HFN and CCBR showed positive expression of CK19 (CK19, $\times 100$ ). (C) Few hair follicles in accessory tragus showed weak expression of CK19 (triangles) (CK19, $\times 100$ ). (D) Hair follicles in trichofolliculoma showed no expression of CK19 (CK19, × 100).

Table 1. Clinical and histopathological features of hair follicle nevus, accessory tragus, cervical chondrocutaneous branchial remnants, and trichofolliculoma

\begin{tabular}{|c|c|c|}
\hline Diagnosis & Clinical features & Histopathological features \\
\hline Hair follicle nevus & $\begin{array}{l}\text { - Usually presents as a skin-colored papule on } \\
\text { the face at birth }\end{array}$ & $\begin{array}{l}\text { - Proliferation of vellus hair follicles with perifollicular } \\
\text { fibrous thickening } \\
\text { - Sebaceous and eccrine glands and smooth muscle fibers } \\
\text { are occasionally seen }{ }^{1,6,7} \\
\text { - No cartilaginous component }\end{array}$ \\
\hline Accessory tragus* & $\begin{array}{l}\text { - Usually presents as a skin-colored papule at birth } \\
\text { - Usually located in the pre-auricular area }\end{array}$ & $\begin{array}{l}\text { - Proliferation of vellus hair follicles with cartilage, prominent } \\
\text { connective tissue framework, and abundant subcutaneous fat }{ }^{1}\end{array}$ \\
\hline $\begin{array}{l}\text { Cervical } \\
\text { chondro-cutaneous } \\
\text { branchial remnants* }\end{array}$ & $\begin{array}{l}\text { - An accessory tragus-like lesion in the cervical } \\
\text { area }^{9}\end{array}$ & - Same as accessory tragus \\
\hline Trichofolliculoma & $\begin{array}{l}\text { - Usually presents as a nodule with central pore } \\
\text { containing hair shafts in adults }{ }^{8}\end{array}$ & $\begin{array}{l}\text { - Central follicular cyst connecting to the peripheral hair } \\
\text { follicles }^{8}\end{array}$ \\
\hline
\end{tabular}

*Cartilaginous components are characteristic features both in accessory tragus and cervical chondrocutaneous branchial remnants, however several cases of accessory tragus without cartilage have been reported.

lesion from the second branchial $\mathrm{arch}^{4}$. According to Davis and Cohen ${ }^{6}$, half of the reported HFN cases presented within the distribution of the first branchial arch. Moreover, several cases of accessory tragus without cartilage have been reported ${ }^{3,4}$. In particular, Asahina et al. ${ }^{4}$ observed a case of multiple accessory tragi without cartilage in the preauricular area and neck. They proposed the concept that HFN and accessory tragus are basically within the same spectrum of hamartomas, although they may differ according to component or location through the developmental process ${ }^{4}$. In line with Asahina et al. ${ }^{4}$, the present case with solitary HFN in the neck also indicates that HFN and accessory tragus are within the same spectrum of disease.

Trichofolliculoma is another lesion that resembles HFN morphologically, which is histologically characterized by a central follicular cyst connecting to the peripheral hair follicles (Fig. 3D, inset). Ackerman et al. ${ }^{10}$ insisted that HFN is actually a trichofolliculoma which includes no central cystic structures because it has been sampled from its periphery. In contrast, other authors still consider HFN as a separate entity from trichofolliculoma, and they sug- 
gest that it is necessary to exam multiple serial sections in order to exclude the presence of central cystic structures ${ }^{8}$. Recently, Karabulut et al. ${ }^{2}$ compared HFN, accessory tragus, and trichofolliculoma from a histopathologic aspect. There was no significant difference among the three diseases in the density of hair follicles, subcutaneous fat score, and presence of connective tissue framework ${ }^{2}$. In the present study, we performed a pilot immunohistochemical study with CK19. CK19, a useful marker for hair follicle stem cell, represents differential potential of hair follicle, and its expression is greater in newborn than older $\operatorname{skin}^{11}$. Misago et al. ${ }^{12}$ observed that CK19 was not expressed in trichofolliculoma, and they proposed that this finding indicates that the hair follicle in trichofolliculoma may not be normal. Consistent with the previous report, in our study hair follicles in trichofolliculoma showed no expression of CK19. In contrast, hair follicles in HFN, accessory tragus, and CCBR expressed CK19 in varying degrees. These findings imply that hair follicles in trichofolliculoma might be different from those in HFN, accessory tragus, and CCBR. Trichofolliculoma seems to result from abortive differentiation of pluripotent skin cells toward hair follicles, whereas HFN, accessory tragus, and CCBR are thought to be hamartomas, overgrowths of mature cells and tissues. However, this study has a limitation in that single cases of each diagnosis were included. Further accumulation of cases is needed to understand more fully the relationship between HFN and differential diagnoses. Varying degrees of CK19 expression in HFN, accessory tragus, and CCBR could be attributed to the different age or location of each lesion.

To our knowledge, this is the first report of HFN solely presenting as a solitary papule on the neck. Our results add to the evidence for HFN, accessory tragus, and CCBR being within the same spectrum of hamartomas and for trichofolliculoma being a separate disease entity from HFN.

\section{CONFLICTS OF INTEREST}

The authors have nothing to disclose.

\section{ORCID}

Ji Su Lee, https://orcid.org/0000-0003-0207-2107
Ji Hoon Yang, https://orcid.org/0000-0002-4271-033X

Hyunsun Park, https://orcid.org/0000-0003-1338-654X

Hyun-Sun Yoon, https://orcid.org/0000-0003-1401-2670

Soyun Cho, https://orcid.org/0000-0003-2468-485X

\section{REFERENCES}

1. Larson KN, O'Shea P, Zedek DC, Morrell DS. Hair follicle nevus located on the chin of an infant: case report and review of literature. Pediatr Dermatol 2016;33:e106-e108.

2. Karabulut YY, Şenel E, Karabulut HH, Dölek Y. Three different clinical faces of the same histopathological entity: hair follicle nevus, trichofolliculoma and accessory tragus. An Bras Dermatol 2015;90:519-522.

3. Nagase K, Nagase K, Misago N, Narisawa Y. A preauricular hairy papule in an infant: hair follicle nevus closely similar to accessory tragus. Arch Dermatol 2012;148:266-268.

4. Asahina A, Mitomi H, Sakurai N, Fujita H. Multiple accessory tragi without cartilage: relationship with hair follicle naevi? Acta Derm Venereol 2009;89:316-317.

5. Jedrych J, Akilov O, Gehris R, Ho J. Hair follicle nevus of the abdominal skin: an unusual extracephalic presentation. Pediatr Dermatol 2014;31:e85-e86.

6. Davis DA, Cohen PR. Hair follicle nevus: case report and review of the literature. Pediatri Dermatol 1996;13:135-138.

7. Serra-Guilléna C, Travesb V, Echeverriaa B, Martorell A. Hair follicle nevus: a case report and review of the literature. Actas Dermosifiliogr 2009; 100:822-824.

8. Motegi S, Amano H, Tamura A, Ishikawa O. Hair follicle nevus in a 2-year old. Pediatr Dermatol 2008;25:60-62.

9. Begovic N, Simic R, Vlahovic A, Kravljanac D, Djuricic $S$, Mijovic T. Cervical chondrocutaneous branchial remnants-report of 17 cases. Int J Pediatr Otorhinolaryngol 2014;78: 1961-1964.

10. Ackerman AB, Viragh PAD, Chongchitnant N. Neoplasms with follicular differentiation. Philadelphia: Lea \& Febiger, 1993. p. xviii-703.

11. Michel M, Török N, Godbout MJ, Lussier M, Gaudreau P, Royal A, et al. Keratin 19 as a biochemical marker of skin stem cells in vivo and in vitro: keratin 19 expressing cells are differentially localized in function of anatomic sites, and their number varies with donor age and culture stage. J Cell Sci 1996;109( Pt 5):1017-1028.

12. Misago N, Kimura T, Toda S, Mori T, Narisawa Y. A revaluation of trichofolliculoma: the histopathological and immunohistochemical features. Am J Dermatopathol 2010;32:3543. 